Pacific Journal of Mathematics

LAGRANGE INVERSION OVER FINITE FIELDS 


\section{LAGRANGE INVERSION OVER FINITE FIELDS}

\section{JOHN GREENE}

A finite field analogue of the Lagrange inversion formula is given and applications to the derivation of character sum identities are discussed.

1. Introduction. In this paper we discuss character sum analogues for Lagrange inversion in one or several variables over finite fields. We then use these techniques as tools for deriving character sum identities. We begin with a short description of classical Lagrange inversion.

If $f(z)$ and $g(z)$ are formal power series where $g(0)=0$ but $g^{\prime}(0) \neq 0$, the inversion problem is to write $f$ as a power series in the variable $g(z)$. The Lagrange inversion theorem gives the solution to this problem. Specifically, the result is

$$
f(z)=f(0)+\sum_{k=1}^{\infty} c_{k} g(z)^{k},
$$

where

$$
c_{k}=\operatorname{Res}_{z} \frac{f^{\prime}(z)}{k g(z)^{k}},
$$

or alternatively,

$$
c_{k}=\operatorname{Res}_{k} \frac{f(z) g^{\prime}(z)}{g(z)^{k+1}} .
$$

Simple proofs of these results can be found in [7] or [10]. These references also contain multivariable generalizations.

Recently there has been much work in developing $q$-analogues for Lagrange inversion, for example, see [1], [3] or [4]. Lagrange inversion is useful in special functions and comes up frequently in deriving transformations and summation theorems as in [5] or [6].

This paper is organized as follows. Theorems analogous to Lagrange inversion are derived in $\$ 2$. The strengths and weaknesses of the analogy are discussed in $\S 3$. In $\$ 4$ we derive several character sum identities from these theorems. We set notation in the remainder of this section. 
Throughout this paper, $G F(q)$ is the finite field with $q=p^{n}$ elements, where $p$ is an odd prime. The capital letters $A, B, L, M, N$ and $R$ and Greek letters $\chi$ and $\theta$ will denote arbitrary multiplicative characters of $G F(q)$. The quadratic character will be denoted by $\varphi$ and the trivial character by $\varepsilon$. All multiplicative characters are defined to be 0 at the 0 element of $G F(q)$. Define $\bar{A}$ by $A \bar{A}=\varepsilon$. We define a function $\delta$ on $G F(q)$ by

$$
\delta(x)= \begin{cases}1, & \text { if } x=0, \\ 0, & \text { if } x \neq 0,\end{cases}
$$

and on multiplicative characters of $G F(q)$ by

$$
\delta(A)= \begin{cases}1, & \text { if } A=\varepsilon, \\ 0, & \text { if } A \neq \varepsilon .\end{cases}
$$

Note that $\delta(x)=1-\varepsilon(x)$. Write $\sum_{x}$ to denote the sum over all $x \in G F(q)$ and $\Sigma_{\chi}$ to denote the sum over all multiplicative characters of $G F(q)$. Let $\zeta=e^{2 \pi l / p}$ and set Tr equal to the trace map from $G F(q)$ to $G F(p)$.

We will make use of the orthogonality relations $[9$, pp. 89, 90]

$$
\begin{gathered}
\sum_{x} \chi(x)=(q-1) \delta(\chi), \\
\sum_{\chi} \chi(x)=(q-1) \delta(1-x),
\end{gathered}
$$

and

$$
\sum_{x} \zeta^{\operatorname{Tr}(x y)}=q \delta(y) .
$$

The Gauss sum of a multiplicative character $A$ is defined by

$$
G(A)=\sum_{x} A(x) \xi^{\operatorname{Tr}(x)},
$$

and the Jacobi sum of $A$ and $B$ is defined by

$$
J(A, B)=\sum_{x} A(x) B(1-x) .
$$

Finally, some easy changes of variables in (1.9) imply

$$
J(A, B)=J(B, A)
$$

and

$$
J(A, B)=B(-1) J(\overline{A B}, B) .
$$

2. Inversion over finite fields. Suppose $\left\{f_{1}(x), \ldots, f_{q}(x)\right\}$ is an orthonormal basis for the vector space of all complex valued functions over $G F(q)$ with respect to the inner product

$$
\langle f(x), g(x)\rangle=\sum_{x} f(x) \overline{g(x)} .
$$


Thus,

$$
\sum_{x} f_{i}(x) f_{j}(x)= \begin{cases}1, & \text { if } i=j \\ 0, & \text { if } i \neq j\end{cases}
$$

Let $M$ be the $q \times q$ matrix $\left(f_{i}\left(x_{j}\right)\right)_{i j}$. Then (2.2) implies $M M^{*}=I$, where $M^{*}$ is the conjugate transpose of $M$. Consequently, $M^{*} M=I$ from which it follows that

$$
\sum_{k=1}^{q} f_{k}(x) \overline{f_{k}(y)}=\delta(x-y) .
$$

If $f(x)$ is any function from $G F(q)$ to $\mathbf{C}$, the orthogonality relations (2.2) and (2.3) imply

$$
f(x)=\sum_{k=1}^{q} c_{k} f_{k}(x),
$$

where

$$
c_{k}=\sum_{x} f(x) \overline{f_{k}(x)}
$$

LEMMA 2.6. Let $f(x): G F(q) \rightarrow \mathrm{C}$ and $g(x): G F(q) \rightarrow G F(q)$. Then for fixed $x$,

$$
\sum f(y)=\sum_{k=1}^{q} c_{k} f_{k}(g(x))
$$

where

$$
c_{k}=\sum_{y} f(y) \overline{f_{k}(g(y))}
$$

and the sum on the left hand side extends over all y such that $g(y)=g(x)$.

Proof. With $c_{k}$ defined as above we have

$$
\begin{aligned}
\sum_{k=1}^{q} c_{k} f_{k}(g(x)) & =\sum_{k=1}^{q} \sum_{y} f(y) \overline{f_{k}(g(y))} f_{k}(g(x)) \\
& =\sum_{y} f(y) \delta(g(x)-g(y))=\sum_{\substack{y: \\
g(y)=}} f(x)
\end{aligned}
$$

as desired.

The two classical examples of orthogonal bases for complex valued functions over $G F(q)$ are the set of all multiplicative characters together with $\delta(x)$, and the set of all additive characters. That these are in fact 
orthogonal bases follows from (1.5) and (1.7). These sets can be made orthonormal by appropriate scaling so we have the following as corollaries to Lemma 2.6.

TheOrEM 2.7. Given $f(x): G F(q) \rightarrow \mathbf{C}$ and $g(x): G F(q) \rightarrow G F(q)$,

(a)

$$
\sum_{\substack{y: \\ g(y)=g(x)}} f(y)=\delta(g(x)) \sum_{\substack{y: \\ g(y)=0}} f(y)+\sum_{\chi} c_{\chi} \chi(g(x)),
$$

where

$$
c_{\chi}=(1 /(q-1)) \sum_{y} f(y) \bar{\chi}(g(y))
$$

(b)

$$
\sum_{\substack{y: \\ g(y)=g(x)}} f(y)=\sum_{y} c_{y} \zeta^{\operatorname{Tr}(g(x) y)},
$$

where $c_{1}=(1 / q) \sum_{x} f(x) \xi^{-\operatorname{Tr}(g(x) y)}$.

The generalization to several variables causes no problems. For example, in the case of functions of two variables it is clear that arguments similar to those in Lemma 2.6 will show the following.

THEOREM 2.8. If $f(x, y): G F(q)^{2} \rightarrow \mathbf{C}$ and $g(x, y), h(x, y): G F(q)^{2}$ $\rightarrow G F(q)$, then

$$
\varepsilon(g(x, y) h(x, y)) \sum f(u, v)=\sum_{\chi, \theta} c_{\chi, \theta} \chi(g(x, y)) \theta(h(x, y)),
$$

where

$$
c_{\chi, \theta}=\frac{1}{(q-1)^{2}} \sum_{x, y} f(x, y) \bar{\chi}(g(x, y)) \bar{\theta}(h(x, y))
$$

and the sum on the left hand side extends over all $(u, v)$ for which $g(u, v)=g(x, y)$ and $h(u, v)=h(x, y)$.

Of course, a similar result holds if the multiplicative characters in Theorem 2.8 are replaced by any orthogonal basis for the complex-valued functions over $G F(q)$.

3. Remarks. There are several drawbacks to Theorems 2.7 and 2.8 . We will discuss them while making more explicit the analogy between Lagrange inversion and the results of $\S 2$. 
The result in (1.2) can be restated

$$
c_{k}=\text { C.T. } z f(z) g^{\prime}(z) g(z)^{-1-k}
$$

where C.T. $f(z)$ is the constant term in the Laurent expansion for $f(z)$. Note that if a function $f(x): G F(q) \rightarrow \mathbf{C}$ is expanded as a character sum,

$$
f(x)=f(0) \delta(x)+\sum_{\chi} c_{\chi} \chi(x)
$$

then

$$
c_{\varepsilon}=\frac{1}{q-1} \sum_{x \neq 0} f(x)
$$

Inspired by this result, define the constant term of the function $f(x)$ by

$$
\text { C.T. } f(x)=\frac{1}{q-1} \sum_{x \neq 0} f(x) \text {. }
$$

With this definition, the constants $c_{\chi}$ in Theorem 2.7(a) are defined by

$$
c_{\chi}=\text { C.T. } f(x) \bar{\chi}(g(x)),
$$

which we contrast with (3.1).

The conditions $g(0)=0, g^{\prime}(0) \neq 0$ in the classical theorem imply that $g(z)$ is one-to-one near $z=0$. If $g(x): G F(q) \rightarrow G F(q)$ is one-to-one, then the sum on the right hand side of Lemma 2.6 reduces to a single term so with $c_{k}$ as in Lemma 2.6, $f(x)=\sum_{k=1}^{q} c_{k} f_{k}(g(x))$. Unfortunately, functions $g: G F(q) \rightarrow G F(q)$ which arise in practice tend not to be one-to-one. Consequently, for most practical problems, the summation on the left hand side of Lemma 2.6 is required.

In the classical theorem, the coefficients $c_{k}$ are unique in the sense that if

$$
\sum_{k=1}^{\infty} c_{k} g(z)^{k}=\sum_{k=1}^{\infty} d_{k} g(z)^{k}
$$

in some neighborhood of $z=0$, then $c_{k}=d_{k}$. The worst drawback to Lemma 2.7 is that the coefficients $c_{k}$ are uniquely determined only if $g(x)$ is one-to-one. If $g(x)$ is not one-to-one, the best that can be said is that if

$$
\sum_{k=1}^{q} c_{k} f_{k}(g(x))=\sum_{k=1}^{q} d_{k} f_{k}(g(x)),
$$

then $c_{k}=d_{k}+h_{k}$ where the coefficients $h_{k}$ satisfy

$$
\sum_{k=1}^{q} h_{k} f_{k}(g(x))=0
$$


for all $x$. For example, if $f(x)=\delta(1-x)$ and $g(x)=x^{2}$, then Theorem 2.7(a) gives

$$
\delta(1-x)+\delta(1+x)=\frac{1}{q-1} \sum_{\chi} \chi\left(x^{2}\right) .
$$

If $a$ is any element of $G F(q)$ which is not a square, then

$$
\frac{1}{q-1} \sum_{\chi} \chi\left(a x^{2}\right)=\delta\left(1-a x^{2}\right)=0
$$

for all $x$. Thus we have

$$
\frac{1}{q-1} \sum_{\chi} \chi\left(x^{2}\right)=\frac{1}{q-1} \sum_{\chi}(1+\chi(a)) \chi\left(x^{2}\right)
$$

for all $x$ in $G F(q)$. The consequence of these remarks is that the classical technique of expanding a function in terms of another function in two different ways and equating coefficients does not generalize well to finite fields.

4. Examples. In this section we give some short examples of Theorem 2.7 and an extended discussion of the uses of Theorem 2.8 in deriving character sum identities.

As a first example, if $f(x)=A(1-x)$ and $g(x)=x$, then

$$
c_{\chi}=\frac{1}{q-1} \sum_{y} A(1-y) \bar{\chi}(y)=\frac{1}{q-1} J(A, \bar{\chi}) .
$$

By Theorem 2.7(a), we have

$$
A(1-x)=\delta(x)+\frac{1}{q-1} \sum_{\chi} J(A, \bar{\chi}) \chi(x) .
$$

This useful result is an analogue for the binomial theorem, which can be made more striking by the introduction of "binomial coefficients" (see [8])

$$
\left(\begin{array}{l}
A \\
B
\end{array}\right)=\frac{B(-1)}{q} J(A, \bar{B}) .
$$

With this definition, (4.1) becomes

$$
A(1-x)=\delta(x)+\frac{q}{q-1} \sum_{\chi}\left(\begin{array}{l}
A \\
\chi
\end{array}\right) \chi(-x) .
$$

From (1.11) we derive a variant of (4.1),

$$
A(1-x)=\delta(x)+\frac{1}{q-1} \sum_{\chi} J(\bar{A} \chi, \bar{\chi}) \chi(-x),
$$


or, in terms of binomial coefficients,

$$
A(1-x)=\delta(x)+\frac{q}{q-1} \sum_{\chi}\left(\begin{array}{c}
\bar{A} \chi \\
\chi
\end{array}\right) \chi(x) .
$$

Examples of the uses of (4.1) and (4.3) can be found in [8].

For an example of Theorem 2.7(b), let $f(x)=\xi^{\operatorname{Tr}(2 x)}$ and $g(x)=x^{2}$. Since $g(x)=g(-x)$ but $x \neq-x$ unless $x=0$,

$$
\sum_{g(y)=g(x)} f(x)=\zeta^{\operatorname{Tr}(2 x)}+\xi^{-\operatorname{Tr}(2 x)}-\delta(x) .
$$

On the right hand side of Theorem 2.7(b) we must calculate

$$
c_{y}=\frac{1}{q} \sum_{x} \zeta^{\operatorname{Tr}\left(2 x-x^{2} y\right)} .
$$

Note that $c_{0}=0$. For $y \neq 0$, replace $x$ by $x+1 / y$ to obtain

$$
\begin{aligned}
c_{y} & =\frac{1}{q} \sum_{x} \zeta^{\operatorname{Tr}\left(1 / y-x^{2} y\right)}=\frac{1}{q} \zeta^{\operatorname{Tr}(1 / y)} \sum_{x} \zeta^{-\operatorname{Tr}\left(x^{2} y\right)} \\
& =\frac{1}{q} \zeta^{\operatorname{Tr}(1 / y)} \sum_{x} \zeta^{-\operatorname{Tr}(x y)}(1+\varphi(x))=\frac{1}{q} \zeta^{\operatorname{Tr}(1 / y)} \sum_{x} \varphi(x) \zeta^{-\operatorname{Tr}(x y)} \\
& =\frac{1}{q} \zeta^{\operatorname{Tr}(1 / y)} G(\varphi) \varphi(-y) .
\end{aligned}
$$

By Theorem 2.7(b) we now have

(4.6) $\zeta^{\operatorname{Tr}(2 x)}+\zeta^{\operatorname{Tr}(2 x)}-\delta(x)=\frac{1}{q} \varphi(-1) G(\varphi) \sum_{y} \varphi(y) \zeta^{\operatorname{Tr}\left(1 / y+x^{2} y\right)}$.

Replacing $y$ by $1 / y$ and using $G(\varphi)^{2}=q \varphi(-1)$ gives

$$
\sum_{y} \varphi(y) \zeta^{\operatorname{Tr}\left(y+x^{2} / y\right)}=G(\varphi)\left(\zeta^{\operatorname{Tr}(2 x)}+\zeta^{\operatorname{Tr}(-2 x)}-\delta(x)\right) .
$$

This is a character sum analogue for

$$
\int_{0}^{\infty} \sqrt{y} e^{-y-x^{2} / y} \frac{d y}{y}=\Gamma\left(\frac{1}{2}\right) e^{-2 x} .
$$

We now derive an identity from Theorem 2.8 and use it to obtain some more substantial character sum formulas. This example was inspired by [6] and follows that paper (by analogy) closely.

Consider functions $f(x, y)$ with the property that $f(x, y)=0$ whenever $x=0, y=0, x=1, y=1$ or $x y=1$. Let

$$
g(x, y)=\frac{x(1-y)}{1-x y} \text { and } h(x, y)=\frac{(1-x) y}{1-x y} .
$$


The mapping

$$
(g(x, y), h(x, y)): G F(q)^{2} \rightarrow G F(q)^{2}
$$

is one-to-one on the set of all $(x, y)$ such that $x y(1-x)(1-y)(1-x y)$ $\neq 0$. Consequently, by Theorem 2.8 we have

$$
f(x, y)=\sum_{\chi, \theta} c_{\chi, \theta} \chi(g(x, y)) \theta(h(x, y)),
$$

where

$$
c_{\chi, \theta}=\frac{1}{(q-1)^{2}} \sum_{u, v} f(u, v) \bar{\chi}(g(u, v)) \bar{\theta}(h(u, v)) .
$$

Also,

$$
g\left(\frac{x}{1-y}, \frac{y}{1-x}\right)=x, \quad h\left(\frac{x}{1-y}, \frac{y}{1-x}\right)=y
$$

so

$$
f\left(\frac{x}{1-y}, \frac{y}{1-x}\right)=\sum_{\chi, \theta} c_{\chi, \theta} \chi(x) \theta(y) .
$$

If we denote

$$
\frac{1}{(q-1)^{2}} \sum_{x, y} f(x, y) \text { by C.T. } f(x, y) \text {, }
$$

then

$$
\begin{aligned}
\text { C.T. } f( & \left.\frac{x}{1-y}, \frac{y}{1-x}\right)=c_{\varepsilon \varepsilon} \\
\quad= & \frac{1}{(q-1)^{2}} \sum_{u, v} f(u, v) \varepsilon(u v(1-u)(1-v)(1-u v)) \\
\quad= & \frac{1}{(q-1)^{2}} \sum_{u, v} f(u, v)=\text { C.T. } f(x, y) .
\end{aligned}
$$

We have proved:

THEOREM 4.9. If $f(x, y): G F(q)^{2} \rightarrow \mathbf{C}$ satisfies $f(x, y)=0$ for all $(x, y)$ such that $x y(1-x)(1-y)(1-x y)=0$, then

$$
\text { C.T. } f\left(\frac{x}{1-y}, \frac{y}{1-x}\right)=\text { C.T. } f(x, y) .
$$

This result is the analogue of the classical result [6, (3)]

$$
\text { C.T. } f\left(\frac{x}{1-y}, \frac{y}{1-x}\right)=\text { C.T. } \frac{1}{1-x y} f(x, y) \text {. }
$$


If we now take $f(x, y)=A(1-x) B(1-y) \bar{L}(x) \bar{M}(y) \overline{A B}(1-x y)$, then

$$
\begin{aligned}
f\left(\frac{x}{1-y}, \frac{y}{1-x}\right)= & \varepsilon(1-x-y) A M(1-x) B L(1-y) \bar{L}(x) \bar{M}(y) \\
= & A M(1-x) B L(1-y) \bar{L}(x) \bar{M}(y) \\
& -\delta(1-x-y) A(1-x) B(x) .
\end{aligned}
$$

By Theorem 4.9 we have

$$
\begin{aligned}
& \sum_{x, y} A(1-x) B(1-y) \bar{L}(x) \bar{M}(y) \overline{A B}(1-x y) \\
&=\sum_{x, y} A M(1-x) B L(1-y) \bar{L}(x) \bar{M}(y) \\
&-\sum_{x, y} \delta(1-x-y) A(1-x) B(x) \\
&= J(A M, \bar{L}) J(B L, \bar{M})-J(A, B) .
\end{aligned}
$$

From (4.4) it follows that

$$
\overline{A B}(1-x y)=\delta(x y)+\frac{1}{q-1} \sum_{\chi} J(A B \chi, \bar{\chi}) \chi(-x y) .
$$

When (4.12) is substituted into the left hand side of (4.11) and the $x$ and $y$ sums are evaluated we have

$$
\begin{gathered}
\frac{1}{q-1} \sum_{\chi} J(A, \bar{L} \chi) J(B, \bar{M} \chi) J(A B \chi, \bar{\chi}) \chi(-1) \\
=J(A M, \bar{L}) J(B L, \bar{M})-J(A, B) .
\end{gathered}
$$

In terms of binomial coefficients,

$$
\frac{q}{q-1} \sum_{\chi}\left(\begin{array}{c}
A \\
L \bar{\chi}
\end{array}\right)\left(\begin{array}{c}
B \\
M \bar{\chi}
\end{array}\right)\left(\begin{array}{c}
A B \chi \\
\chi
\end{array}\right)=\left(\begin{array}{c}
A M \\
L
\end{array}\right)\left(\begin{array}{c}
B L \\
M
\end{array}\right)-\frac{B M L(-1)}{q}\left(\begin{array}{c}
A \\
\bar{B}
\end{array}\right) .
$$

This is an analogue for the binomial coefficient version of Saalschütz's theorem [6, (1)]

$$
\sum_{k \geq 0}\left(\begin{array}{c}
a \\
l-k
\end{array}\right)\left(\begin{array}{c}
b \\
m-k
\end{array}\right)\left(\begin{array}{c}
a+b-k \\
k
\end{array}\right)=\left(\begin{array}{c}
a+m \\
l
\end{array}\right)\left(\begin{array}{c}
b+l \\
m
\end{array}\right)
$$

If we take

$$
f(x, y)=\varepsilon(1-x) \varepsilon(1-y) \bar{L}(x) \bar{M}(y) N(x-y) \bar{N}(1-x y),
$$


then Theorem 4.9 gives

$$
\text { (4.16) } \begin{aligned}
\text { C.T. }(\varepsilon(1-x) \varepsilon(1-y) \bar{L}(x) \bar{M}(y) N(x-y) \bar{N}(1-x y)) \\
=\text { C.T. }(\bar{L}(x) M(1-x) \bar{M}(y) L(1-y) N(x-y) \varepsilon(1-x-y)) .
\end{aligned}
$$

Using $\varepsilon(a)=1-\delta(a)$ and converting (4.16) to a sum we obtain

$$
\begin{aligned}
\sum_{x, y} \bar{L}(x) & M(1-x) \bar{M}(y) L(1-y) N(x-y)-(q-1) \delta(N) \\
= & \sum_{x, y} \bar{L}(x) \bar{M}(y) N(x-y) \bar{N}(1-x y) \\
& -(q-1) \delta(M)-(q-1) N(-1) \delta(L) .
\end{aligned}
$$

Writing $N(x-y)=N(x) N(1-y / x)$ and applying (4.1) to $N(1-y / x)$ and $\bar{N}(1-x y)$, the summation on the right hand side becomes

$$
\frac{1}{(q-1)^{2}} \sum_{x, y, \chi, \theta} J(N, \bar{\chi}) J(\bar{N}, \bar{\theta}) \bar{L} N \bar{\chi} \theta(x) \bar{M} \chi \theta(y) .
$$

The $x$ and $y$ sums are 0 unless $\bar{L} N \bar{\chi} \theta=\varepsilon$ and $\bar{M} \chi \theta=\varepsilon$, so we must have $\theta^{2}=L M \bar{N}$ and $\chi=M \bar{\theta}$. Note $\theta^{2}=(\varphi \theta)^{2}$. We have

$$
\begin{aligned}
& \sum_{x, y} \bar{L}(x) M(1-x) \bar{M}(y) L(1-y) N(x-y) \\
& =(q-1) \delta(N)-(q-1) \delta(M)-(q-1) N(-1) \delta(L) \\
& +\left\{\begin{aligned}
0, & \text { if } L M \bar{N} \text { is not a square, } \\
J(N, \bar{M} R) J(\bar{N}, \bar{R})+J(N, \varphi \bar{M} R) J(\bar{N}, \varphi \bar{R}), & \text { if } L M \bar{N}=R^{2} .
\end{aligned}\right.
\end{aligned}
$$

Similar results to (4.18) can be found in [2, (5)] and [8, 4.37]. If we use (4.1) in the left hand side of (4.18), appeal to properties (1.10) and (1.11) for Jacobi sums and convert Jacobi sums to binomial coefficients, we have

$$
\begin{aligned}
& \frac{q}{q-1} \sum_{\chi}\left(\begin{array}{c}
N \\
\chi
\end{array}\right)\left(\begin{array}{c}
M \\
L \bar{N} \chi
\end{array}\right)\left(\begin{array}{c}
L \\
L \bar{M} \chi
\end{array}\right) \chi(-1) \\
&=\frac{q-1}{q^{2}} L M(-1) \delta(N)-\frac{q-1}{q^{2}} L N(-1) \delta(M) \\
&-\frac{q-1}{q^{2}} M(-1) \delta(L) \\
&+\left(\begin{array}{c}
0, \quad \text { if } L M \bar{N} \text { is not a square, } \\
M R(-1)\left(\begin{array}{c}
N R \\
N
\end{array}\right)\left(\begin{array}{c}
N \\
L \bar{R}
\end{array}\right)+\varphi M R(-1)\left(\begin{array}{c}
\varphi N R \\
N
\end{array}\right)\left(\begin{array}{c}
N \\
\varphi L \bar{R}
\end{array}\right), \\
\text { if } L M \bar{N}=R^{2} .
\end{array}\right.
\end{aligned}
$$


This is an analogue for the binomial coefficient version of Dixon's theorem [6, (2)]

$$
\begin{aligned}
\sum_{k \geq 0}\left(\begin{array}{l}
n \\
k
\end{array}\right)\left(\begin{array}{c}
m \\
l-
\end{array}\right)\left(\begin{array}{c}
l \\
l-m+k
\end{array}\right)(-1)^{k} \\
\quad=\left\{\begin{array}{c}
0, \text { if } l+m-n \text { is odd, } \\
(-1)^{m-r}\left(\begin{array}{c}
n+r \\
n
\end{array}\right)\left(\begin{array}{c}
n \\
l-r
\end{array}\right),
\end{array}\right.
\end{aligned}
$$

Finally, if we take

$$
\begin{aligned}
f(x, y)= & \varepsilon(1-x) \varepsilon(1-y) \bar{L}(x) \bar{M}(y) \bar{N}(1-x y) \\
& \times N(x-\alpha y-(1-\alpha) x y),
\end{aligned}
$$

then

$$
\begin{aligned}
f\left(\frac{x}{1-y},\right. & \left.\frac{y}{1-x}\right) \\
& =\varepsilon(1-x-y) \bar{L}(x) M(1-x) \bar{M}(y) L(1-y) N(x-\alpha y) .
\end{aligned}
$$

If we take $\alpha \neq 0$, then with calculations similar to the previous example,

$$
\begin{aligned}
\text { C.T. } f\left(\frac{x}{1-y}, \frac{y}{1-x}\right) \\
=\frac{L(-1)}{(q-1)^{3}} \sum_{\chi} J(N, \bar{\chi}) J(M, \bar{L} N \bar{\chi}) J(L, \bar{L} M \bar{\chi}) \chi(\alpha) \\
\quad-\frac{q}{(q-1)^{2}} \delta(1+\alpha)-\frac{1}{q-1} \varepsilon(1+\alpha) \delta(N) \\
\quad+\frac{1}{(q-1)^{2}} N(-\alpha)+\frac{1}{(q-1)^{2^{\prime}}}
\end{aligned}
$$

and

$$
\begin{aligned}
\text { C.T. } f(x, y)= & \sum_{\chi} J\left(\overline{L M N} \chi^{2}, \bar{\chi}\right) J(N, \bar{L} M \bar{\chi}) J(\bar{N}, \bar{L} N \bar{\chi}) \chi\left(\frac{\alpha}{(1-\alpha)^{2}}\right) \\
& +c_{2}\left\{\begin{array}{l}
0, \quad \text { if } \bar{L} M N \text { is not a square } \\
J(N, \bar{R}) J(\bar{N}, \bar{M} R)+J(N, \varphi \bar{R}) J(\bar{N}, \varphi \bar{M} R), \\
\quad \text { if } \bar{L} M N=R^{2}
\end{array}\right. \\
& -\frac{1}{q-1} \delta(M)-\frac{N(-\alpha)}{q-1} \delta(L)+\frac{1}{(q-1)^{2}}+\frac{N(-\alpha)}{(q-1)^{2}} \\
& +\frac{1}{(q-1)^{2}} \bar{L} M N\left(\frac{-\alpha}{1-\alpha}\right) J(\bar{M}, \bar{N})
\end{aligned}
$$


where

$$
c_{1}=\frac{N(-1)}{(q-1)^{3}} \bar{L} M N(1-\alpha)
$$

and

$$
c_{2}=\frac{L(-1)}{(q-1)^{2}} \delta(1-\alpha) .
$$

By Theorem 4.9, after converting Jacobi sums to binomial coefficients,

$$
\begin{aligned}
\frac{q}{q-1} \sum_{\chi}\left(\begin{array}{c}
N \\
\chi
\end{array}\right)\left(\begin{array}{c}
M \\
L \bar{N} \chi
\end{array}\right)\left(\begin{array}{c}
L \\
L \bar{M} \chi
\end{array}\right) \chi(-\alpha) \\
=E_{1}+E_{2}+L N(-1) \bar{L} M N(1-\alpha) \frac{q}{q-1} \\
\quad \times \sum_{\chi}\left(\begin{array}{c}
L \overline{M N} \chi^{2} \\
\chi
\end{array}\right)\left(\begin{array}{c}
N \\
L \bar{M} \chi
\end{array}\right)\left(\begin{array}{c}
\bar{N} \\
L \bar{N} \chi
\end{array}\right) \chi\left(\frac{\alpha}{(1-\alpha)^{2}}\right)
\end{aligned}
$$

where $E_{1}$ and $E_{2}$ are the "error" terms

$$
\begin{aligned}
E_{1}= & \frac{q-1}{q^{2}} L M(-1) \varepsilon(1+\alpha) \delta(N) \\
& -\frac{q-1}{q^{2}} L N(-1) \delta(M)-\frac{q-1}{q^{2}} M(-1) N(\alpha) \delta(L) \\
& +\frac{L M N(-1)}{q} \delta(1+\alpha)+\frac{1}{q^{2}} \bar{L} M N\left(\frac{\alpha}{1-\alpha}\right) J(\bar{M}, \bar{N})
\end{aligned}
$$

and

$$
E_{2}=\delta(1-\alpha)\left\{\begin{array}{l}
0, \quad \text { if } L M \bar{N} \text { is not a square } \\
M R(-1)\left(\begin{array}{c}
N R \\
N
\end{array}\right)\left(\begin{array}{c}
N \\
L \bar{R}
\end{array}\right)+\varphi M R(-1)\left(\begin{array}{c}
\varphi N R \\
N
\end{array}\right)\left(\begin{array}{c}
N \\
\varphi L \bar{R}
\end{array}\right) \\
\text { if } L M \bar{N}=R^{2} .
\end{array}\right.
$$

When $\alpha=1$, this reduces to (4.15). For general $\alpha,(4.17)$ is an analogue for the following version of Whipple's ${ }_{3} F_{2}$-quadratic transformation [6, (11)]

(4.22) $\sum_{k \geq 0}\left(\begin{array}{l}n \\ k\end{array}\right)\left(\begin{array}{c}l \\ l-m+k\end{array}\right)\left(\begin{array}{c}l \\ l-m-k\end{array}\right)(-\alpha)^{k}$

$$
\begin{aligned}
= & (1-\alpha)^{m+n-l} \\
& \times \sum_{J \leq(m+n-l) / 2} \frac{(l+j) !}{j !(l-n+j) !(l-n+j) !(m+n-l-2 j) !}\left(\frac{-\alpha}{(1-\alpha)^{2}}\right)^{\prime} .
\end{aligned}
$$


The identities (4.14), (4.19) and (4.21) indicate that there is an analogue for hypergeometric series over finite fields. In fact, [8] describes such an analogue in which these results are proved, by different methods, as hypergeometric series identities.

\section{REFERENCES}

[1] G. E. Andrews, Identities in combinatorics II: A q-analog of the Lagrange inversion theorem, Proc. Amer. Math. Soc., 53 (1975), 240-245.

[2] R. Evans, Identities for products of Gauss sums over finite fields, L'Enseignement Math., 27 (1981), 197-209.

[3] A. Garsia, A q-analogue of the Lagrange inversion formula, Houston J. Math., 7 (1981), 205-237.

[4] I. Gessel, A noncommutative generalization and q-analogue of the Lagrange inversion formula, Trans. Amer. Math. Soc., 257 (1980), 455-482.

[5] I. Gessel and D. Stanton, Strange evaluations of hypergeometric series, SIAM J. Math. Anal., 13 (1982), 295-308.

[6] Short proofs of Saalschütz's and Dixon's theorems, J. Combinatorial Theory Ser. A, 38 (1985), 87-90.

[7] I. J. Good, Generalization to several variables of Lagrange's expansion with applications to stochastic processes, Proc. Cambridge Philos. Soc., 56 (1960), 367-380.

[8] J. Greene, Hypergeometric functions over finite fields, Trans. Amer. Math. Soc., 301 (1987), 77-101.

[9] K. Ireland and M. Rosen, A classical Introduction to Modern Number Theory, Springer-Verlag, New York, 1982.

[10] C. Jacobi, De resolution aequationum per series infinitas, J. Reine und Angew. Math., 6 (1830), 257-286.

Received June 20, 1986.

SOUTHERN ILLINOIS UNIVERSITY

CARbondale, IL 62901 



\section{EDITORS}

\author{
V. S. VARADARAJAN \\ (Managing Editor) \\ University of California \\ Los Angeles, CA 90024 \\ HeRbert Clemens \\ University of Utah \\ Salt Lake City, UT 84112 \\ R. FINN \\ Stanford University \\ Stanford, CA 94305
}

\author{
HERMANN FLASCHKA \\ University of Arizona \\ Tucson, AZ 85721 \\ RAMESh A. GANGOLLI \\ University of Washington \\ Seattle, WA 98195 \\ VAUGHAN F. R. JONES \\ University of California \\ Berkeley, CA 94720
}

\author{
ROBION KIRBY \\ University of California \\ Berkeley, CA 94720 \\ C. C. MOORE \\ University of California \\ Berkeley, CA 94720 \\ HAROLD STARK \\ University of California, San Diego \\ La Jolla, CA 92093
}

\section{ASSOCIATE EDITORS}
R. ARENS
E. F. BECKENBACH
B. H. NEUMANN
F. WOLF
K. YOSHIDA
(1906-1982)

\section{SUPPORTING INSTITUTIONS}

UNIVERSITY OF ARIZONA
UNIVERSITY OF BRITISH COLUMBIA
CALIFORNIA INSTITUTE OF TECHNOLOGY
UNIVERSITY OF CALIFORNIA
MONTANA STATE UNIVERSITY
UNIVERSITY OF NEVADA, RENO
NEW MEXICO STATE UNIVERSITY
OREGON STATE UNIVERSITY

\author{
UNIVERSITY OF OREGON \\ UNIVERSITY OF SOUTHERN CALIFORNIA \\ STANFORD UNIVERSITY \\ UNIVERSITY OF HAWAII \\ UNIVERSITY OF TOKYO \\ UNIVERSITY OF UTAH \\ WASHINGTON STATE UNIVERSITY \\ UNIVERSITY OF WASHINGTON
}

The Supporting Institutions listed above contribute to the cost of publication of this Journal, but they are not owners or publishers and have no responsibility for its content or policies.

Mathematical papers intended for publication in the Pacific Journal of Mathematics should be in typed form or offset-reproduced (not dittoed), double spaced with large margins. Please do not use built up fractions in the text of the manuscript. However, you may use them in the displayed equations. Underline Greek letters in red, German in green, and script in blue. The first paragraph must be capable of being used separately as a synopsis of the entire paper. In particular it should contain no bibliographic references. Please propose a heading for the odd numbered pages of less than 35 characters. Manuscripts, in triplicate, may be sent to any one of the editors. Please classify according to the scheme of Math. Reviews, Index to Vol. 39. Supply name and address of author to whom proofs should be sent. All other communications should be addressed to the managing editor, or Elaine Barth, University of California, Los Angeles, California 90024.

There are page-charges associated with articles appearing in the Pacific Journal of Mathematics. These charges are expected to be paid by the author's University, Government Agency or Company. If the author or authors do not have access to such Institutional support these charges are waived. Single authors will receive 50 free reprints; joint authors will receive a total of 100 free reprints. Additional copies may be obtained at cost in multiples of 50 .

The Pacific Journal of Mathematics is issued monthly as of January 1966. Regular subscription rate: $\$ 190.00$ a year (5 Vols., 10 issues). Special rate: $\$ 95.00$ a year to individual members of supporting institutions.

Subscriptions, orders for numbers issued in the last three calendar years, and changes of address should be sent to Pacific Journal of Mathematics, P.O. Box 969, Carmel Valley, CA 93924, U.S.A. Old back numbers obtainable from Kraus Periodicals Co., Route 100, Millwood, NY 10546.

The Pacific Journal of Mathematics at P.O. Box 969, Carmel Valley, CA 93924 (ISSN 0030-8730) publishes 5 volumes per year. Application to mail at Second-class postage rates is pending at Carmel Valley, California, and additional mailing offices. Postmaster: send address changes to Pacific Journal of Mathematics, P.O. Box 969, Carmel Valley, CA 93924.

PUBLISHED BY PACIFIC JOURNAL OF MATHEMATICS, A NON-PROFIT CORPORATION Copyright (c) 1987 by Pacific Journal of Mathematics 


\section{Pacific Journal of Mathematics}

\section{Vol. 130, No. $2 \quad$ October, 1987}

Bernhard Banaschewski, J. L. Frith and C. R. A. Gilmour, On the congruence lattice of a frame ............................ 209

Paul S. Bourdon, Density of the polynomials in Bergman spaces ........2215

Lawrence Jay Corwin, Approximation of prime elements in division

algebras over local fields and unitary representations of the multiplicative group

Stephen R. Doty and John Brendan Sullivan, On the geometry of extensions of irreducible modules for simple algebraic groups

Karl Heinz Dovermann and Reinhard Schultz, Surgery of involutions

with middle-dimensional fixed point set ....................2 275

Ian Graham, Intrinsic measures and holomorphic retracts ............ 299

John Robert Greene, Lagrange inversion over finite fields . . . . . . . . . . . 313

Kristina Dale Hansen, Restriction to $\mathrm{GL}_{2}(\mathrm{O})$ of supercuspidal

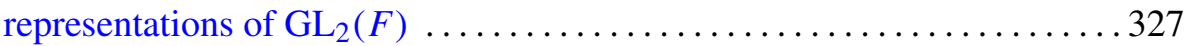

Kei Ji Izuchi, Unitary equivalence of invariant subspaces in the polydisk ....351

A. Papadopoulos and R. C. Penner, A characterization of pseudo-Anosov

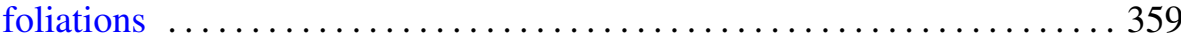

Erik A. van Doorn, The indeterminate rate problem for birth-death

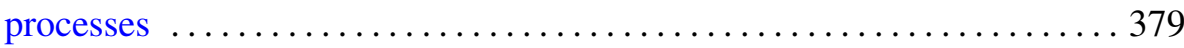

Ralph Jay De Laubenfels, Correction to: "Well-behaved derivations on

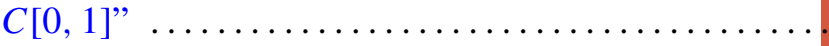

Robert P. Kaufman, Correction to: "Plane curves and removable sets" . . . . 396

Richard Scott Pierce and Charles Irvin Vinsonhaler, Correction to:

"Realizing central division algebras" 